\title{
Identification of QTLs for Yield and Drought- Related Traits in Maize: Assessment of Their Causal Relationships
}

Ana Nikolić, Dragana Ignjatović-Micić, Dejan Dodig, Violeta Anđelković \& Vesna Lazić-Jančić

To cite this article: Ana Nikolić, Dragana Ignjatović-Micić, Dejan Dodig, Violeta Anđelković \& Vesna Lazić-Jančić (2012) Identification of QTLs for Yield and Drought-Related Traits in Maize: Assessment of Their Causal Relationships, Biotechnology \& Biotechnological Equipment, 26:3, 2952-2960, DOI: 10.5504/BBEQ.2012.0016

To link to this article: https://doi.org/10.5504/BBEQ.2012.0016

(c) 2012 Taylor and Francis Group, LLC

曲 Published online: 16 Apr 2014.

Submit your article to this journal $\widetilde{ }$

Ull Article views: 364

4 Citing articles: 4 View citing articles 준 


\title{
IDENTIFICATION OF QTLS FOR YIELD AND DROUGHT-RELATED TRAITS IN MAIZE: ASSESSMENT OF THEIR CAUSAL RELATIONSHIPS
}

\author{
Ana Nikolić, Dragana Ignjatović-Micić, Dejan Dodig, Violeta Anđelković, Vesna Lazić-Jančić \\ Maize Research Institute "Zemun Polje”, Belgrade, Serbia \\ Correspondence to: Ana Nikolić \\ E-mail: anikolic@mrizp.rs
}

\begin{abstract}
Drought is one of the most important factors contributing to crop yield loss. Developing maize varieties with drought tolerance requires exploring the genetic basis of yield and associated agronomic traits. In order to identify QTLs for yield and drought related traits and to determine their relationships, $116 F_{3}$ families of the DTP79 $\times B 73$ cross were evaluated in field trials. Phenotypic correlations between the traits were calculated using Pearson's coefficient. QTL detection was performed using ANOVA and composite interval mapping (CIM). Out of 64 QTLs identified using CIM for all analyzed traits 26 QTLs were also identified with ANOVA. For all the traits (except for EL, DS2 and RWC1) only a small proportion of phenotypic variation was accounted for by the identified QTL. The significant positive/negative phenotypic correlations between different traits and coincidence of markers with allelic differences in the expected direction show that they may be causally correlated. The above criteria were fullfilled for a subset of QTLs within all the traits. The overlap found between the QTLs for yield and those for the other traits suggests the presence of genes with pleiotropic effects on the investigated traits. Results will be used for further investigation and detection of possible candidate genes for drought tolerance in maize.
\end{abstract}

Biotechnol. \& Biotechnol. Eq. 2012, 26(3), 2952-2960

Keywords: maize, drought tolerance, grain yield, drought tolerance traits, phenotypic correlations, QTLs, additive effect

\section{Introduction}

Water availability is the most important limiting factor for crop production worldwide. It has been estimated that approximately US $\$ 10$ billion of primary food production is lost annually because of insufficient rainfall (21). Global warming and increased unpredictability of the intensity and frequency of rainfall patterns underline the urgency and need for a more effective improvement of crop yield under drought conditions. Maize is the third most important crop for food production after rice and wheat. Due to the growing demand for dairy and meat products in developing countries and the decline in rice production in China and India, maize has been projected to become the most important crop by 2030 (21). Also, the importance of maize goes beynd food and feed, as maize today is one of the major biofuel and biotech crops in the world $(23,28)$.

Traditionally, increasing maize yield under drought through direct selection for yield has been restricted by its low heritability and large "genotype $\times$ environment" interaction. Still, morpho-physiological traits correlated with yield and displaying increased genetic variability in drought conditions have been commonly identified and selected for in maize breeding programs (6). Morpho-physiological and other socalled secondary traits (such as anthesis-silking interval (ASI), leaf senescence, leaf erectness, rolling, osmotic potential, ears per plant, kernels per ear, kernels per plant, etc.) can be used for indirect selection under drought conditions $(4,5)$.
Development of molecular genetics has given new opportunities for drought tolerance improvement. Quantitative genetics, with wide range of molecular markers now available, provide opportunities, not only for the identification of the genetic factors (quantitative trait loci-QTLs) determining the phenotypic value of a particular trait, but also for the analysis of relationships between traits $(12,25)$. By comparing the coincidence of QTLs for specific traits and QTLs for yield under drought, it is possible to test whether a particular constitutive or adaptive response to drought stress is significant in improving field level drought tolerance. For two traits to be causally related, the significant QTL effect of one trait should have a measurable effect on the other trait. Coincidence of QTL for two traits, with allelic difference corresponding to the expected causal relationship between traits, is strong evidence that the two traits are related $(17,27)$.

Quantitative trait analysis is being used to test the relationships between physiological traits in many crops, such as maize (12), potato (25) and Stylosanthes scabra (27). Quarrie et al. (18) used QTL analysis for establishing relationships between physiological and morphological traits across cereals. In maize, mainly experiments on relationships between $\mathrm{ABA}$, yield and other drought related traits have been conducted $(12,18,22)$.

Drought tolerant population (DTP) originally developed by CIMMYT is being used for more than ten years as a source of drought tolerance in commercial breeding programs. One of the inbreed lines extracted from this population was used with the aim to identify the QTLs associated to yield and yield components, different morphological and physiological traits under drought and to study the causal nature of these 
relationships through QTLs analysis. The implications of the results for development of high yielding drought tolerant maize hybrids are discussed.

\section{Materials and Methods}

\section{Plant materials and field trial}

A total of $116 \mathrm{~F}_{3}$ families derived from the cross of DTP79 (drought tolerant maize line extracted from DTP developed by CIMMYT) and B73 (drought susceptible maize line) were used to make an $\mathrm{F}_{2}$ mapping population. Field data were obtained from trials with $\mathrm{F}_{3}$ families, which were produced by selfing $\mathrm{F}_{2}$ plants. The field trial was conducted in 2007 at

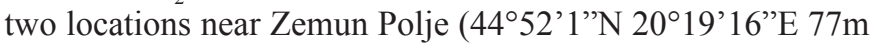
a.s.), Serbia. A three-replicate randomised block design was used in the trial. Planting was performed on the optimum date (mid April) and plots consisted of single rows at $70 \mathrm{~cm}$ spacing with a total of 20 plants per row after thinning. Conventional cultural practices were applied in all test plots.

\section{Phenotypic measurements}

Grain yield (GY) was measured in grams per plant. Measurements of the number of kernels per row (NKR), number of rows per ear (NRE), and ear length (EL) were taken on ten randomly chosen plants from all three replicas. The same ten plants per family used for GY, were also scored for anthesissilking interval (ASI) and visually-scored drought score (DS). To determine ASI the date of pollen shedding (when more than $50 \%$ of chosen plants showed anthers extruded from the tassel glums) and the date of silking (when more than 50\% of plants showed silks extruded from the ear husks) were recorded. ASI was computed as the difference between days to siliking and pollen sheding. DS was visually scored, using a scale from 1 (plants with leaves severely wilted, yellow and rolled) to 5 (plants with fully turgid leaves, green and unrolled leaves). DS was recorded regularly within the following two weeks at 5 p.m. Recording was performed for the first time on $24^{\text {th }}$ July (DS1) at 5 p.m. when the maximum air temperature was $43.6{ }^{\circ} \mathrm{C}$ (the highest temperature ever measured at Zemun Polje, Belgrade, Serbia). Four measurements were conducted on rainy days within this period, and their mean is given as DS2 (recording performed on $30^{\text {th }}$ and $31^{\text {st }} \mathrm{July}$, and on $2^{\text {nd }}$ and $5^{\text {th }}$ August). For the rest of this period, the mean of the measurements is given as DS3 (Fig. 1).

Measurements for physiological traits such as relative water content (RWC), osmotic potential (OP) and relative sugar content (RSC) were done on five out of the ten plants, during the flowering period. Leaf discs for assessing RWC were collected twice, on $17^{\text {th }}$ July (RWC1), and one week later - on $24^{\text {th }}$ July (RWC2). After sampling in the field, fresh weight of the bulked samples of each family was measured. Samples were placed in Petri dishes containing distilled water, at $4{ }^{\circ} \mathrm{C}$, for 3 hours. The leaf samples were then blotted gently between filter papers, and turgid weight was recorded. The same samples were then oven dried overnight at $80^{\circ} \mathrm{C}$, in order to obtain dry weight. Leaf relative water content was estimated using the following formula:

$R W C(\%)=100 \times($ Fresh weight - Dry weight $) /($ Turgid weight - Dry weight)

Leaf samples for measuring OP and RSC taken together with samples for assessing RWC, were frozen and then thawed prior to measurements. Cell sap was expressed from the leaf discs after thawing using a screw press (19) and its solute concetration measured using a freezing point depression osmometer (Roebling, Camlab, Cambrige, UK). Leaf osmotic potential was calculated by the formula: $O P(M P a)=c \times R$ $\times T$, where $c$ is the solute concetration (milliosmoles), $R$ $\left(8.32 \mathrm{~J} \cdot \mathrm{mol}^{-1} \cdot \mathrm{K}^{-1}\right)$ is the gas constant, and $T(\mathrm{~K})$, the absolute temperature of the leaf when sampled, assumed to be 298 K. Relative sugar content (\%) was determined using optical refractometer, from leaf juice already squeezed for measuring osmotic potential.

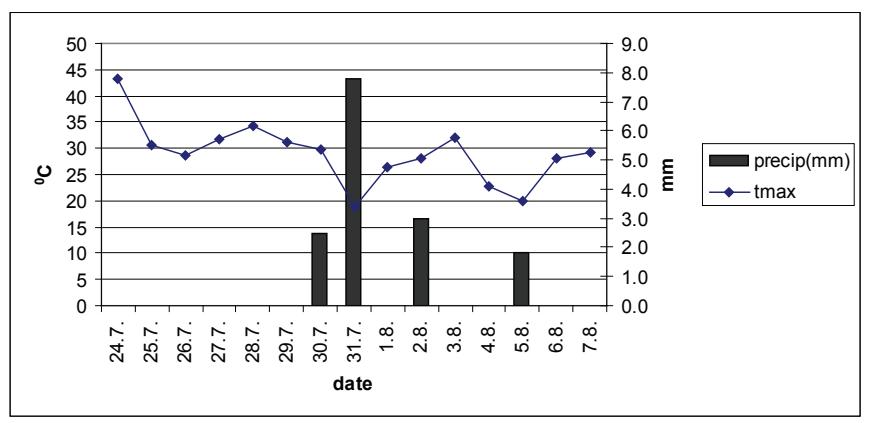

Fig. 1. Climate diagram.

Correlations between analyzed traits using row data were calculated with Pearson correlation coefficient.

\section{Molecular marker analysis}

A linkage map based on $\mathrm{F}_{2}$ population was constructed using MAPMAKER (11) with Haldane function (10). The population was genotyped by restriction fragment length polymorphism (RFLP), simple-sequence repeats (SSR), and amplified fragment length polymorphism (AFLP) markers. The map was made with a total of 234 markers, of which the 200 most informative markers were used for QTL analysis.

Initially, to detect possible QTL, simple one-way ANOVA was carried out. A significant F-ratio for genotype mean squares for a particular locus was taken to indicate the possible presence of a QTL. Significance level used for QTL detection was $\mathrm{P}<0.1$.

QTL analysis was also performed using WinQTL Cartographer, 2.5 version software (http://statgen.ncsu.edu). For QTL identification with WinQTL Cartographer Composite interval mapping (CIM) methods $(32,33)$ were used. A putative QTL was declared significant when the LOD score was $>2.0$. Although a LOD threshold of 2.0 implies a high chance for experiment-wise Type-I error (that one or more of the QTLs represent a false positive), this value was chosen based on the following considerations: 1) it allows more complete comparison with published results on QTLs for the traits in 
drought stressed maize obtained with a LOD treshold of 2.0;2) simulation work (3) indicated that Type-II errors (not declaring the presence of a real QTL) represent a greater problem than Type-I errors, particularly when a population of limited size is considered, as in the present work (22). The maximum LOD score along the interval was taken as the position of the QTL (the QTL peak), and the confidence interval of each QTL is the one-LOD support interval, which is determined by finding the region on both sides of a QTL peak that corresponds to a decrease of 1 LOD score. QTL additive effects were calculated according to Sanguineti et al. (22).

\section{Results and Discussion}

\section{Phenotypic relationships between the traits}

The Pearson correlation coefficients $(r)$ between investigated traits in $116 \mathrm{~F}_{3}$ maize families are presented in Table 1. The grain yield was positively correlated with $\operatorname{NKR}(r=0.64 * * *)$, NRE $\left(r=0.60^{* * *}\right)$, EL $\left(r=0.37^{* *}\right)$, DS2 $\left(r=0.33^{* *}\right)$, DS3 $(r$ $\left.=0.29^{* *}\right)$ and $\operatorname{RSC} 1(r=0.22 *)$. On the other hand, there was high negative correlation between GY and ASI $(r=-0.62 * * *)$. While yield components and drought scores were positively correlated with GY, there was no correlation between GY and physiological traits (RWC, OP and RSC), with the exception of RSC1.

The anthesis-silking interval was negatively correlated with yield components and drought scores. There was no significant correlation between ASI and physiological traits, except for RWC2 $\left(r=-0.30^{* *}\right)$. Yield components (NKR, NRE and EL) were higly correlated $(\mathrm{P}<0.001)$, as expected. A significant association between yield components and drought scores was obtained for EL with DS2 $\left(r=0.41^{* * *}\right)$, EL with DS3 $(r=$ $\left.0.41^{* * *}\right)$, NRE with DS2 $\left(r=0.23^{*}\right)$ and NRE with DS3 $(r$ $\left.=0.23^{*}\right)$. All three drought score measurements were highly correlated $(\mathrm{P}<0.001)$.

The relationship of physiological traits with drought scores in most cases was non-significant, except between RWC2 and DS1 $\left(r=0.27^{* *}\right)$ as well as between RSC2 and DS3 $(r$ $=-0.23 *)$. Several significant correlations were also found between physiological traits. The highest ones were between $\operatorname{RSC} 2$ and $\mathrm{OP} 2(r=0.79 * *), \operatorname{RSC} 1$ and $\operatorname{RSC} 2(r=0.58 * * *)$ as well as $\mathrm{RSC} 1$ and $\mathrm{OP} 1\left(r=0.54^{* * *}\right)$.

\section{QTL analysis}

QTL mapping methods include single point analysis (looks for QTL on the marker itself) and interval mapping (detects QTL at regular intervals between two flanking markers). Although the most precise data on QTLs are obtained with interval mapping, this method needs suitable markers and large populations with sufficient number of recombinants. For this reason we first performed one-way ANOVA (single point analysis) and then CIM (interval mapping with additional markers between the flanking markers, as cofactors in the analysis) using LOD > 2.0 .

For marker assisted selection (MAS) application in breeding programmes, QTL detected with higher stringency
$(\mathrm{P}<0.01$ and $\mathrm{LOD}<2.4)$ may be used to reduce the number of false positives. However, this may result in the loss of potential QTL through false negatives. Out of 64 QTLs identified using CIM for all analyzed traits, 26 QTLs were also identified with ANOVA. Fifteen of these 26 QTLs were detected when the probability level was equal to or greater than $\mathrm{P}<0.05$. When the probability level was reduced to $\mathrm{P}<0.1$ another 11 putative QTLs were found. Recent studies in tomato have shown that some of the QTL detected with low stringency $(\mathrm{P}<0.1)$ were consistently detected across different environments while some of the QTL detected with high stringency $(\mathrm{P}<0.01)$ were not detected in all the environments (9). Therefore, these QTLs obtained with low stringency may be treated as potential QTL subject to further testing. Similar significance levels were used by other authors $(7,12,16,25,27)$.

QTLs identified by both methods (ANOVA and CIM) are all QTLs for GY and DS3 and five out of six QTLs were identified for DS2. For most of the other traits, only one or no QTLs were matched. Although QTLs identified by both ANOVA and CIM could have the greatest influence on the expression of the analyzed traits, due to the small size of the analyzed population QTLs identified by either of the methods could also have significant impact on the trait and should not be excluded from further experiments.

The total number of detected significant QTLs using CIM for each trait, chromosome positions, $L R\left(L O D=L R / 2 \mathrm{Ln}_{10}\right)$ values, additive effect and percentage of phenotypic variation explained by the QTL are reported in Table 2, and their additive effects are given in Table 3. Illustration of all identified QTLs is given in Fig. 2.

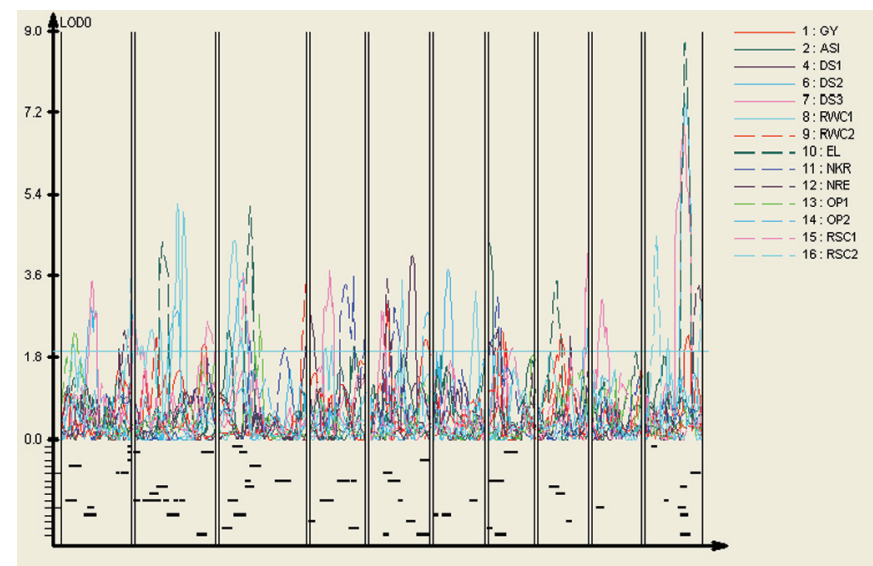

Fig. 2. Illustration of identified QTLs on all 10 chromosomes obtained by WinQTLChart 2.5.

As the total genome was not fully covered by the markers (e.g. chromosome 8), all the QTL associated with a trait might not have been detected in the present study. Moreover, the proportion of QTL identified for a trait could be estimated with some loss of precision. The small population size would inevitably result in overestimation of the amount of variance accounted for by QTL, but at least this may give an approximation of the QTL not detected (27). For most traits 
TABLE 1

Pearson correlation coefficient matrix for the different maize traits

\begin{tabular}{|c|c|c|c|c|c|c|c|c|c|c|c|c|c|c|}
\hline Trait & GY & ASI & DS1 & DS2 & DS3 & EL & NKR & NRE & RWC1 & RWC2 & OP1 & OP2 & RSC1 & RSC2 \\
\hline GY & - & & & & & & & & & & & & & \\
\hline ASI & $-0.62 * * *$ & - & & & & & & & & & & & & \\
\hline DS1 & 0.15 & $-0.22 *$ & - & & & & & & & & & & & \\
\hline DS3 & $0.29 * *$ & $-0.38 * * *$ & $0.46^{* * *}$ & 0.90 *** & - & & & & & & & & & \\
\hline EL & $0.37 * * *$ & -0.18 & 0.21 & $0.41 * * *$ & $0.39^{* * *}$ & - & & & & & & & & \\
\hline NKR & $0.64 * * *$ & $-0.40 * *$ & 0.06 & 0.12 & 0.12 & $0.29 * *$ & - & & & & & & & \\
\hline RWC1 & 0.01 & \begin{tabular}{|l|}
-0.01 \\
\end{tabular} & -0.03 & 0.02 & \begin{tabular}{|l|}
-0.002 \\
\end{tabular} & -0.06 & 0.04 & \begin{tabular}{|l|}
-0.02 \\
\end{tabular} & - & & & & & \\
\hline RWC2 & 0.11 & $-0.30 * *$ & $0.27 *$ & 0.20 & 0.18 & 0.12 & 0.03 & 0.12 & 0.12 & . & & & & \\
\hline OP1 & 0.15 & 0.15 & 0.12 & 0.02 & 0.01 & -0.02 & 0.14 & 0.07 & -0.20 & 0.04 & - & & & \\
\hline OP2 & -0.07 & 0.00 & -0.04 & -0.18 & -0.20 & -0.04 & 0.1 & -0.02 & -0.19 & 0.06 & $0.31 * *$ & - & & \\
\hline RSC1 & $0.22 *$ & -0.13 & 0.07 & 0.01 & 0.05 & 0.06 & 0.14 & 0.14 & $-0.22 *$ & 0.05 & $0.54 * * *$ & $0.49 * * *$ & - & \\
\hline RSC2 & 0.07 & -0.001 & -0.06 & -0.21 & $-0.23 *$ & -0.09 & 0.09 & -0.01 & -0.20 & 0.00 & $0.32 * *$ & $0.79 * * *$ & $0.58 * * *$ & - \\
\hline
\end{tabular}

GY: grain yield;ASI: anthesis-silking interval; DS1: drought score on 24 ${ }^{\text {th }}$ July; DS2: drought score after rain; DS3: drought score in the afternoon; EL: ear lenght; NKR: number of kernels per row; NRE: number of rows per ear; RWC1, RWC2: relative water content; OP1, OP2: osmotic potential; SC1, RSC2: relative sugar content; $*, * *, * * *$ Significantly different at $\mathrm{P}<0.05, \mathrm{P}<0.01$ and $\mathrm{P}<0.001$, respectively.

TABLE 2

Intervals containing QTL with a LOD score of $>2.0^{*}$

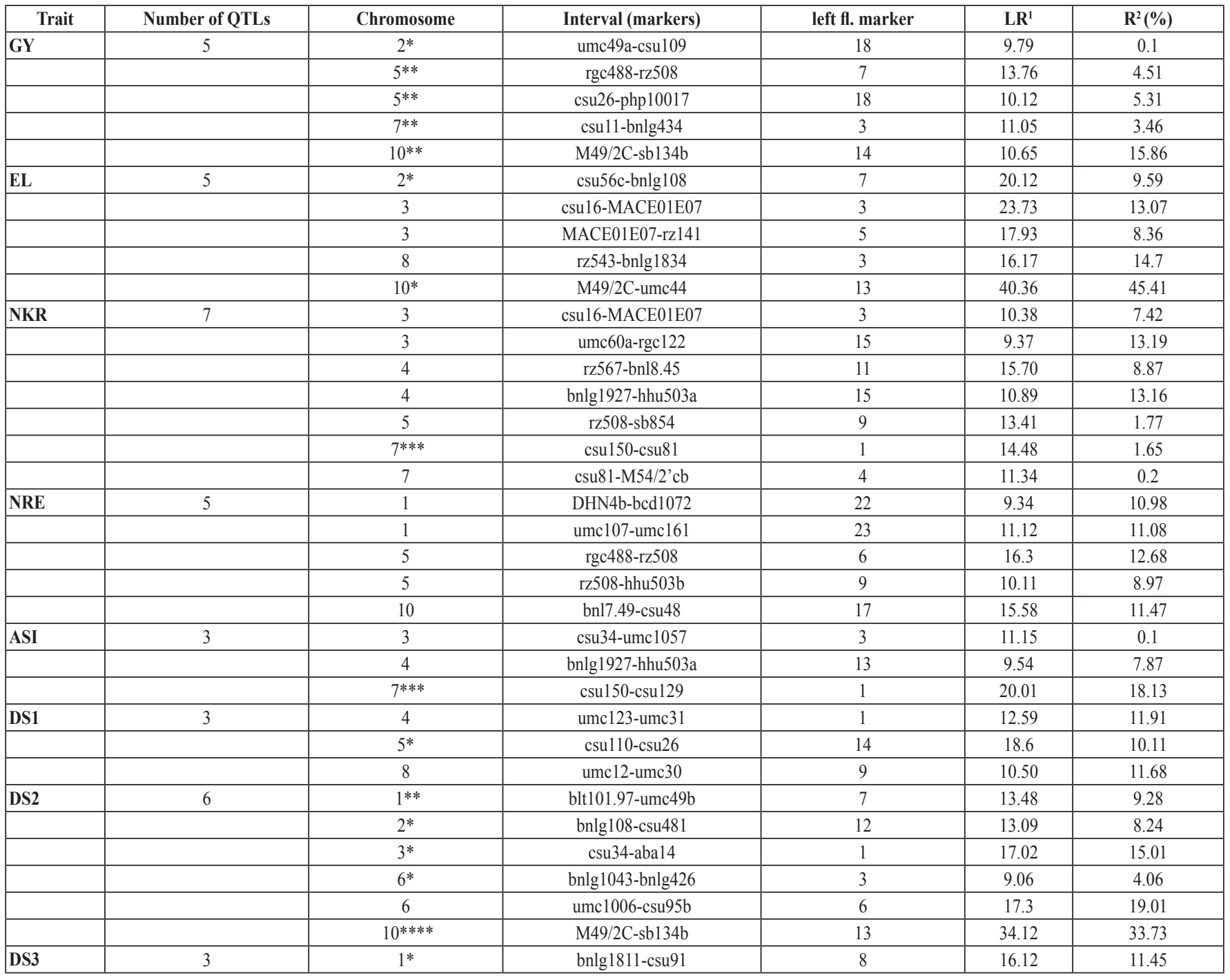




\begin{tabular}{|c|c|c|c|c|c|c|}
\hline & & 9* & csu95a-bnlg1372 & 3 & 14.24 & 0.1 \\
\hline & & $10^{* *}$ & bnlg1074-umc44 & 12 & 31.98 & 26.55 \\
\hline \multirow[t]{12}{*}{ RWC1 } & 12 & 1 & bnlg1014-M52/1B & 3 & 9.98 & 2.94 \\
\hline & & $2 * * *$ & umc53-sb134a & 1 & 11.31 & 11.98 \\
\hline & & 2 & bnlg1327-umc34 & 3 & 9.68 & 16.21 \\
\hline & & 2 & csu56c-umc5 & 4 & 11.32 & 3.02 \\
\hline & & 2 & M54/2'Ca-bnlg108 & 11 & 10.42 & 0.8 \\
\hline & & 2 & bcd855-csu481 & 12 & 23.96 & 69.32 \\
\hline & & 3 & csu34-umc1057 & 1 & 20.28 & 69.4 \\
\hline & & 4 & rz630-rgc390a & 3 & 9.44 & 18.99 \\
\hline & & 4 & rgc390a-psr128b & 7 & 9.71 & 13.48 \\
\hline & & 5 & sb854-csu173 & 12 & 16.29 & 61.37 \\
\hline & & $6 * *$ & sb851a-umc62 & 16 & 15.16 & 8.46 \\
\hline & & $10^{* *}$ & hhu504a-cdo456 & 9 & 10.21 & 64.86 \\
\hline \multirow[t]{2}{*}{ RWC2 } & 2 & 2 & umc61-csu46 & 5 & 10.38 & 4.66 \\
\hline & & 8 & bnlg1834-csu155 & 5 & 10.89 & 0.31 \\
\hline \multirow[t]{3}{*}{ OP1 } & 3 & 1 & umc157-umc11 & 3 & 11.25 & 8.85 \\
\hline & & $3 * *$ & MACE01A07-csu68c & 7 & 9.92 & 11.82 \\
\hline & & 3 & umc60b-sb160b & 10 & 12.72 & 8.73 \\
\hline \multirow[t]{2}{*}{ OP2 } & 2 & $1 *$ & umc161-sb663b & 26 & 14.06 & 3.45 \\
\hline & & 5 & csu227-php10017 & 18 & 12.97 & 2.47 \\
\hline \multirow[t]{5}{*}{ RSC1 } & 5 & 1 & umc161-sb663b & 27 & 13.59 & 6.83 \\
\hline & & $2 * *$ & umc53-sb134a & 1 & 10.82 & -0.31 \\
\hline & & $2 *$ & bnlg1520-csu166 & 18 & 12.15 & 5.68 \\
\hline & & 3 & umc1057-aba14 & 1 & 16.59 & 7.94 \\
\hline & & 7 & bnlg434-bnl1407 & 8 & 9.37 & 12.47 \\
\hline \multirow[t]{3}{*}{\begin{tabular}{|l|} 
RSC2 \\
\end{tabular}} & 3 & 1 & umc161-bnlg2331 & 25 & 16.32 & 9.72 \\
\hline & & 3 & csu34-csu16 & 1 & 10.99 & 1.1 \\
\hline & & $10^{* * *}$ & csu103a-sb113 & 3 & 20.75 & \\
\hline
\end{tabular}

"Abbreviations as per Table 1.

TABLE 3

Additive effects of the overlapping QTLs of the analyzed traits*

\begin{tabular}{|c|c|c|c|c|c|c|c|c|c|c|c|c|c|c|}
\hline Ch. Number & GY & EL & NKR & NRE & ASI & DS1 & DS2 & DS3 & RWC1 & RWC2 & RSC1 & RSC2 & OP1 & OP2 \\
\hline 1 & & & & & & & 0.20 & 0.20 & & & & & & \\
\hline 1 & & & & & & & & & & & 0.45 & 0.64 & & 18.23 \\
\hline 1 & & & & & & & & & -2.25 & & & & -26.54 & \\
\hline 2 & & & & & & & & & 4.95 & 2.29 & & & & \\
\hline 2 & -1.8 & & & & & & & & & & \begin{tabular}{|l|}
-0.39 \\
\end{tabular} & & & \\
\hline 2 & & & & & & & 0.19 & & -1.68 & & & & & \\
\hline 3 & & & & & -0.1 & & & & -16.71 & & & & & \\
\hline 3 & & & & & & & 0.24 & & & & 0.53 & & & \\
\hline 3 & & & & & & & 0.24 & & -16.71 & & & 0.21 & & \\
\hline 3 & & 1.29 & -0.9 & & & & & & & & 0.53 & & & \\
\hline 3 & & 0.97 & & & & & & & & & & & -30.65 & \\
\hline 5 & & & -0.42 & & & & & & -25.71 & & & & & \\
\hline 5 & 11.3 & & & & & & & & & & & & & 33.12 \\
\hline 7 & 10.48 & & $0.42 / 0.16$ & & & & & & & & & & & \\
\hline 7 & & & 0.42 & & -1.19 & & & & & & & & & \\
\hline 7 & 10.48 & & & & & & & & & & 0.58 & & & \\
\hline 8 & & -1.31 & & & & & & & -0.65 & & & & & \\
\hline 10 & 21.99 & 2.72 & & & & & 0.37 & 0.29 & & & & & & \\
\hline
\end{tabular}

"Abbreviations as per Table 1. 
only a small proportion of phenotypic variation was accounted for by the identified QTL. The only exceptions are EL, DS2 and RWC1.

\section{Coincidence between QTLs for yield and yield components}

Five grain yield QTLs were identified using CIM - one per chromosomes 2, 7 and 10, and two on chromosome 5. All QTLs detected with Cartographer were also significant with ANOVA-QTLs on chromosomes 5, 7 and 10 were significant at $\mathrm{P}<0.05$, while one on chromosome 2 was significant at $\mathrm{P}<0.1$. The percentage of variation explained by these QTLs ranged from 0.1 to $15.86 \%$.

Seventeen QTLs affecting yield components were identified using CIM. Among them five QTLs influenced EL (one per chromosomes 2, 8 and 10 and two on chromosome 3), five NRE (chromosomes 1 and 5 contained two QTLs and chromosome 10, one QTL) and seven NKR (chromosomes 3, 4 and 7 with two QTLs, and chromosome 10 with one QTL). Two QTLs for EL on chromosomes 2 and 10 and one QTL for NKR on chromosome 7 were significant with ANOVA at $\mathrm{P}<0.1$ and $\mathrm{P}<0.01$, respectively. The phenotypic variance explained by these QTLs ranged from 8.36 to $45.41 \%$ for EL, from 0.2 to $13.19 \%$ for NKR, and from 8.97 to $12.68 \%$ for NRE.

Regions of one QTL for GY and one QTL for NRE overlapped on chromosome 5. Also, chromosome region harbouring coinciding QTLs affecting GY and NKR were found on chromosome 7. And finally, coincident QTL for GY and EL was found on chromosome 10. Considering QTL coincidence between yield components EL and NKR QTLs partially overlapped on chromosome 3, while QTL affecting NKR partially overlapped with the second QTL for NRE on chromosome 5 .

The additive effects for yield and all three yield components were positively associated, in expected directions (Table 3). Additive effects of EL and NKR on chromosome 3 were in opposite direction, although highly significant positive phenotypic correlation was determined between these two traits.

\section{Coincidence between QTLs for yield, yield components and ASI, drought score}

Three QTLs for ASI using CIM were identified on chromosomes 3, 4 and 7. Only one QTL on chromosome 7 was significant by ANOVA $(\mathrm{P}<0.01)$. Individual QTL accounted for $0.1-18.13 \%$ of the phenotypic variation. No common QTLs were found for GY and ASI. QTL for ASI on chromosome 4 completely and QTL on chromosome 7 partially overlapped with QTLs for NKR. Additive effects for ASI and NKR on chromosomes 4 and 7 were in the expected directions.

The number of QTLs detected for drought score through CIM was as follows: three for DS1 (on chromosomes 4, 5 and 8), six for DS2 (one per chromosomes 1,2, 3 and 10, and two on chromosome 6) and three for DS3 (on chromosomes
1, 9 and 10). Only one QTL for DS1 on chromosome 5 was significant with ANOVA $(\mathrm{P}<0.1)$. ANOVA identified five significant QTLs for DS2 on chromosomes $1(\mathrm{P}<0.05), 2$ $(\mathrm{P}<0.1), 3(\mathrm{P}<0.1), 6(\mathrm{P}<0.1)$ and $10(\mathrm{P}<0.001)$. All QTLs detected with CIM for DS3 were also observed with ANOVA $(\mathrm{P}<0.1$ on chromosomes 1 and $9 ; \mathrm{P}<0.05$ on chromosome 10). The range of explained QTL phenotypic variance for these traits was from 10.11 to $11.91 \%$ for DS1, from 4.06 to $33.73 \%$ for DS2, and from 0.1 to $26.55 \%$ for DS3. QTL for EL on chromosome 2 partially overlapped with QTL for DS2. The region that contains coinciding QTLs for GY, EL, DS2 and DS3 was detected on chromosome 10. The additive effects of all the traits were in the expected direction.

\section{Coincidence between QTLs for yield, yield components and physiological traits}

Relative water content (RWC), osmotic potential (OP) and relative sugar content (RSC) were measured twice and subjected to QTL analysis. A total of 14 QTLs for RWC were detected on all chromosomes except chromosomes 7,8 and 9. For RWC1 the number of identified QTLs was 12 (one per chromosomes 1, 3, 5, 6 and 10, two on chromosome 4, and five on chromosome 2), while only two QTLs were identified for RWC2 (on chromosomes 2 and 5). QTLs for RWC1 were significant by ANOVA on chromosomes $2(\mathrm{P}<0.01), 6$ $(\mathrm{P}<0.05)$, and $10(\mathrm{P}<0.05)$. There were no QTLs for RWC2 significant by ANOVA. The percentage of variation explained by the QTLs ranged from 0.8 to $69.4 \%$ for RWC1 and from 0.31 to $4.66 \%$ for RWC2.

Five QTLs altogether were identified for OP. Chromosome regions containing QTLs for OP1 and OP2 were located on chromosomes 1 and 3 (one on chromosome 1 and two on chromosome 3), and on chromosomes 1 and 5, respectively. For OP1 and OP2 QTLs on chromosome $3(\mathrm{P}<0.05)$ and on chromosome $1(\mathrm{P}<0.1)$ were detected using ANOVA. The percentage of variation explained by the QTLs ranged from 8.73 to $11.82 \%$ for OP1 and from 2.47 to $3.45 \%$ for OP2. Finally, eight QTLs for RSC were identified on chromosomes 1, 2, 3, 7 and 10. There were five QTLs for RSC1 (one per chromosomes 1, 3 and 7 and two on chromosome 2) and three QTLs for RSC2 (on chromosomes 1, 3 and 10). All these QTLs were identified using CIM. QTLs for RSC1 were significant by ANOVA on chromosomes $2(\mathrm{P}<0.05), 3(\mathrm{P}<0.1)$ and for RSC2 $10(\mathrm{P}<0.05)$. The percentage of variation explained by the QTLs ranged from 5.68 to $12.47 \%$ for RSC1 and from 1.1 to $14.54 \%$ for RSC2.

QTLs for GY overlapped with QTLs for RSC1 on chromosomes 2 and 7 (partially) and with QTL for OP2 on chromosome 5. The additive effects for GY and RSC1 were in the expected directions on both chromosomes, but not for GY and OP2. QTLs for NKR partially overlapped with QTL for RSC1 on chromosome 3. The additive efects for these two traits were not in the expected directions. QTL for EL completely overlapped with two QTLs for RWC1 (also with a DS2 QTL) and with one QTL for RWC2 on chromosome 2. 
Another QTL for EL on chromosome 8 overlapped with the QTL for RWC2. The additive effects for EL and RWC were in the expected directions on both chromosomes. Also, one QTL for EL overlapped with one QTL for OP1 on chromosome 3. The additive effects for these two traits were also in the expected directions.

\section{Coincidence of QTLs between physiological traits and ASI and drought score}

Chromosome 1 contained one region with overlapping QTLs for OP1 and RWC1, and another region with overlapping QTLs for OP2, RSC1 and RSC2.

The abovementioned QTL for DS2, which partially overlapped with QTL for EL and one QTL for RWC1 in one region, completely overlapped with another QTL for RWC1 on chromosome 2 . In the first case additive effects for RWC1 and DS2 QTLs were in the expected directions, but not in the second case. RWC1 QTL which partially overlapped with QTL for DS2 also overllaped with the EL QTL. On the same chromosome another QTL for RWC1 overlapped with the QTL for RSC1. The additive effects for these two QTLs were not in the expected direction.

Chromosome 3 contained one QTL for RWC1 which partially overlapped with QTLs for DS2 and RSC2 in one region, and in the other region the same QTL partially overlapped with the QTL for ASI. The additive effects for DS2 and RSC2 were in the expected directions, while for RWC1, DS2 and RSC2, as well as for RWC1 and ASI they were not in the expected directions. On the same chromosome QTL for RSC1 partially overlapped with QTL for DS2, and the additive effect was in the expected direction.

\section{Causal relationships between traits}

The significant positive/negative correlations between different traits using phenotypic data and coincidence of markes with allelic differences in the expected direction show that they may be causally correlated. Coincidence of QTL for two traits with QTL effects in the expected direction may not provide conclusive, but it provides circumstantial evidence that two traits are causally related. Isolation of the genes underlying the traits may provide ultimate evidence that the two traits are causally related (27). In contemplating relationships of examined traits in the context of plant response to drought stress, it should be pointed out that particular measurements of physiologal traits, i.e. DS1, RWC2, OP2 and SC2, were performed on $24^{\text {th }}$ July - the warmest day ever measured in Serbia. The values of these measurements could be treated as plant reaction to shock stress conditions.

The above criteria were fullfilled for a subset of QTLs within all the traits. Between yield and yield components and among yield components phenotypic correlatons were positive and highly significant along with some QTLs overlapping. Also, the additive effects of these QTLs were in the expected directions, except for EL and NKR on chromosome 3. The regions of QTL for GY, EL, DS2 and DS3 overlapped only on chromosome 10. Their additive effects were in the expected directions, and the analysis of phenotypic correlations showed that they were highly positively correlated. ASI and NKR were highly negatively correlated. The regions for QTLs for these two traits overlapped on chromosomes 4 and 7, and the additive effects were also in the expected directions.

A significant coefficient of correlation between yield and physiological traits was detected only for GY and SC1, and coincident QTLs for these two traits were found on chromosomes 2 and 7, with additive effects in the expected directions. Regarding the physiological traits, coincident QTL were found for OP1, RSC1 and RSC2, the additive effects were in the expected directions, and these traits were positively significantly correlated. On chromosome 2 QTL for RWC1 and RSC1 overlapped, with additive effects in the expected directions. RWC decrease and sugar content increase in drought stressed plants was previously observed $(8,26$, $30)$. This is in accordance with the significant and negative correlation between RWC and RSC detected in our study.

The overlap found between the QTLs for yield and those for the other traits suggests the presence of genes with pleiotropic effects on the investigated traits.

\section{Comparison between the QTLs}

In order to check the identified QTLs, i.e. to find out if these QTLs were expressed in different genetic backgrounds and environments, comparison between the QTLs observed in this study and these published in other papers was performed.

QTLs for yield, drought score, RWC, OP and SC have also been identified in another maize study carried out on $\mathrm{F}_{3}$ families derived from the same cross used in this experiment (DTP79 and B73) (S.A. Quarrie, personal communication). Both analysis detected common chromosomal regions harbouring QTLs for the same traits. QTL positioned on chromosome 5 for yield was found in the same region where two yield QTLs were identified in the previous study (Quarrie et al., personal communication). Also, regions for yield QTLs identified on chromosome 7 and 10 in this study coincided with regions for yield QTLs identified by Quarrie et al. (personal communication). However, superior alleles detection was not consistent in all cases. For example, yield QTLs on chromosomes 5 and 10 displayed B73 alleles in the work of Quarrie et al. (personal communication) but DTP79 alleles in our work as superior ones. However, in both experiments yield QTL on chromosome 7 showed DTP79 alleles as superiors.

Chromosome 1 contained one region where QTLs for drought score were detected in both studies and B73 contributed alleles in either case. Also, the region for DS QTL identified by Quarrie et al. (personal communication) partially overlapped with a region for DS1 on chromosome 4 and B73 alleles were superior in both cases. Two QTLs for RWC1 on chromosome 2 were identified on the same position as QTLs for RWC in a prevoius study (Quarrie et al., personal communication) and DTP79 contributed alleles in either case. Regions for RWC QTLs (RWC2 in this study) on chromosome 8 detected in this and previous study (Quarrie et al., personal communication) 
partially overlapped and showed contribution of DTP79 alleles. Common region for OP QTL was also detected on chromosome 1 (OP1 in our study). For this QTL, a contribution of DTP79 alleles was found in this study, in comparison to the previous one (Quarrie et al., personal communication) in which the contributed alleles were from B73. Identification of the same QTLs important for stress tolerance, inspite of differences between the experiments, could indicate that the factors identified represent genetic components with significant impact on traits expression. However, QTLs identified only in one of the studies could be missed in the other study due to different experimental conditions and population size.

QTLs identified in this study were also previously identified by other authors. The same QTLs for yield were identified on 2.09 bin by Beavis (3) and on chromosome 5 by Agrama et al. (1). QTL for yield on chromosome 7 in our study was detected in the region between $7.2-7.03$ bins, while several authors detected QTL for yield between 7.04 - 7.05 bins (2, 3, 13, 20). QTL for ASI identified on 4.07 bin was also identified by Messmer et al. (14) under drought stress condition. Positions of QTLs for NRE found in this study coincided with regions of QTL for this trait previously identified on chromosomes 1 and 10 (2). Three QTLs for RWC on chromosome 2 identified in this study partially overlapped with already mapped QTLs for this trait (22).

The identification of QTLs is only a first step of a longer process aimed at identifying and isolating the underlying molecular cause at the sequence level of the functional variation revealed by QTL analysis. After identification of a (major) QTL, the next step is to identify the most sutable candidate sequence, validate its role, and proceed accordingly with a more direct manipulation of the target trait. The identificaton of candidate genes and the elucidation of their role can be greatly facilitated by combining QTL analysis with different sources of information and technological platforms $(15,24,29,31)$. In this respect recent progress in high-throuhput profiling of the transcriptome, proteome and metabolome enables the investigatation of the concerted expression of thousands of genes and measurement of the level of their products. Considering this, the results from this study will be used for further investigation and detection of possible candidate genes for drought tolerance in maize.

\section{Conclusions}

Yield stability under drought is the most imporatant issue in developing new maize hybrdis with better performance under drought. Considering the low heritability of yield and high genotype $\times$ environment interaction and limited possibility to increase maize yield under drought through direct selection, other traits (e.g. ASI) should be used in the proceses of creating drought tolerant maize genotypies. In this context, identified QTLs in our work, especially those detected in other experiments and further research will help in elucidating the genetic basis of drought tolerance and enable more effective breeding for drought tolerant maize hybrids.

\section{Acknowledgements}

The authors would like to thank Prof. Steve Quarrie and Prof. Sofija Pekic Quarrie for the idea and their contributions to the experiment.

\section{REFERENCES}

1. Agrama H.A.S., Moussa M.E. (1996) Euphytica, 91, 8997.

2. Austin D.F., Lee M. (1996) Theor. Appl. Genet., 92, 817826.

3. Beavis W.D. (1994) In: Proceedings of the 49th Annual Corn and Sorghum Research Conference (D.B. Wilkinson, Ed.), Chicago, Illinois, Washington DC, USA, American Seed Trade Association, 250-266.

4. Bolanos J., Edmeades G.O., Martinez L. (1993) Field Crops Res., 31, 269-286.

5. Bolanos J., Edmeades G.O. (1996) Field Crops Res., 48, 65-80.

6. Campos H., Cooper M., Habben J.E., Edmeades G.O., Schussler J.R. (2004) Field Crops Res., 90, 19-34.

7. Davies P.J., Simko I., Mueller S.M,. Yencho G.C., Lewis C., Mcmurry S., Taylor M.A., Ewing E.E. (1999) Physiol. Plant., 106, 210-218.

8. Dekanova K., Luxova M., Gasparikova O., Kolarovic L. (2004) Biologia, 59(Suppl. 13), 151-155.

9. Fulton T.M., Gradillo S., Beck-Bunn T., Fridman E., Frampton A., Lopez J., Petiard V., Uhlig J., Zamir D., Tanksley S.D. (2000) Theor. Appl. Genet., 100, 10251042.

10. Haldane J.B.S. (1919) J. Genet., 8, 299-309.

11. Lander E.S., Green P., Abrahamson J., Barlow A., Daly M.J., Lincoln S.E., Newburg L. (1987) Genomics, 1, 174-181.

12. Lebreton C., Lazic-Jancic V., Steed A., Pekic S., Quarrie S.A. (1995) J. Exp. Bot., 46, 853-865.

13. Melchinger A.E., Utz H., Schon C.C. (1998) Genetics, 149, 383-403.

14. Mesmmer R., Fracheboud Y., Bazinger M., Vargas M., Stamp P., Ribaut J.M. (2009) Theor. Appl. Genet., 119(5), 913-930.

15. Pflieger S., Lefebvre V., Causse M. (2001) Mol. Breed., 7, 275-291.

16. Prioul J.L., Quarrie S.A., Causse M., De Vienne D. (1997) J. Exp. Bot., 48, 1151-1163.

17. Quarrie S.A. (1996) Plant Growth Regul., 20, 167-178.

18. Quarrie S.A, Laurie D.A., Zhu J., Lebreton C., Semikhoskii A., Steed A., Witsenboer H., Calestani C. (1997) Plant Mol. Biol., 35, 155-165.

19. Quarrie S.A., Henson I.E. (1982) Zeitschrift fur Pflanzenphysiologie, 108, 365-373. 
20. Ribaut J.M., Jiang C., Gonzalez de Leon D. et al. (1997) Theor. Appl. Genet., 94, 887-896.

21. Salvi S., Sponza G., Morgante M. et al. (2007) Proc. Natl. Acad. Sci. USA, 104, 11376-11381.

22. Sanguineti M.C., Tuberosa R., Landi P., Salvi S., Maccaferi M., Casarini E., Conti S. (1999) J. Exp. Bot., 337, 1289-1297.

23. Schwietzke S., Kim Y., Ximenez E., Mosier N., Ladisch M. (2009) In: Molecular Genetic Approaches to Maize Improvement (A.L. Kriz, B.A. Larkins, Eds.), SpringerVerlag, Berlin, Heidelberg, 347-364.

24. Sharp R.E., Poroyko V., Hejlek L.G., Spollen W.G., Springer G.K., Bohnert H.J., Nguyen H.T. (2004) J. Exp. Bot., 55, 2343-2351.

25. Simko I., Mcmurry S., Yang H.M., Manschot A., Davies P.J., Ewing E.E. (1997) Plant Physiol., 115, 1453-1459.

26. Thomas H., James A.R. (1999) New Phytol., 142, 295305.
27. Thumma B.R., Naidu B.P., Chandra A., Cameron D.F., Bahnisch L.M., Liu C. (2001) J. Exp. Bot., 52, 203-214.

28. Torney F., Moeller L., Scarpa A., Wang K. (2007) Curr. Opin. Biotech., 18, 193-199.

29. Tuberosa R., Gill B.S., Quarrie S.A. (2002) Plant Mol. Biol., 48, 445-449.

30. Valentovic P., Luxova M., Kolarovic L., Gasparikova O. (2006) Plant Soil Environ., 52(4), 186-191.

31. Wayne M.L., Mcintyre L. (2002) Proc. Natl. Acad. Sci. USA, 99, 14903-14906.

32. Zeng Z.B. (1993) Proc. Natl. Acad. Sci. USA, 90, 1097210976.

33. Zeng Z.B. (1994) Genetics, 136, 1457-1468. 